6

7 (1) São Carlos Institute of Chemistry, University of São Paulo, P.O. Box 780, CEP 13560-970, 8 São Carlos, SP, Brazil

$14 *$ Corresponding author

15 Phone: +55 1633739932

16 E-mail: artur@iqsc.usp.br

\section{electrolyte \\ Coupling ultrasound to the electro-oxidation of methyl paraben synthetic wastewater: effect of frequency and supporting} D. Dionisio ${ }^{1,2}$, A.J. Motheo ${ }^{1, *}$, C. Sáez², M.A. Rodrigo ${ }^{2}$ 6 (2) Department of Chemical Engineering, Faculty of Chemical Sciences \& Technologies, Universidad de Castilla - La Mancha, Campus Universitario s/n, 13071 Ciudad Real, Spain 


\section{Abstract}

18 In this paper, single electrolysis with diamond electrode is studied and compared to its coupling with sonolysis for the degradation of methyl paraben (MeP). Complete mineralization of MeP was achieved for both processes, in chloride and sulfate media. Results showed that, although the oxidation of pollutant is faster in the presence of chloride, sulfate medium favored the mineralization. Ultrasound irradiation enhanced the removal of organic matter due to the activation of oxidant species in both supporting electrolytes. Moreover, the formation of $\mathrm{Cl}_{2}$ in chloride medium, improves the ultrasound cavitation effect, promoting faster depletion of TOC (total organic carbon) in the first hour of treatment. Regarding the formation of more toxic products, all possible organochlorinated intermediates were removed and chlorates and perchlorates only start to be produced after total removal of TOC. Additionally, the coupling to ultrasound accelerates the destruction of intermediates and delays the formation of $\mathrm{ClO}_{3}{ }^{-}$ and $\mathrm{ClO}_{4}{ }^{-}$. Low and high ultrasound frequencies were evaluated and were found to produce different effects of cavitation, which affect the electrolysis in different ways. The final result will be a balance between those effects and, thus, an optimal frequency may be observed for distinctive systems.

\section{$34 \quad$ Keywords}

35 Methyl paraben, diamond anodes, electrolysis, sonolysis

\section{Highlights}

38 - Complete mineralization of methyl paraben is achieved in sulfate and chloride media;

39 - Sulfate medium favored the removal of TOC;

40 - Enhancement of electrolysis by the coupling to sonolytic process;

41 - Cavitation effects of low and high intensities are balanced in an optimal frequency;

42 - Low frequency ultrasound promotes the activation of persulfate. 
44

Mehyl paraben (MeP) is one of a series of preservatives, which are esters of $p$-hydroxybenzoic acid and include among others, methyl, ethyl, propyl, butyl and benzyl parabens. This compound is widely used in pharmaceutical drugs, cosmetics and personal care products, singly or in combination to other preservatives, in order to achieve the intended antimicrobial effect [1, 2]. In 2004, parabens were firstly related to human breast cancer by Darbre et al. [3], fact that was further confirmed by Dagher et al. in 2012 [4]. Since the first publication, numerous studies have been reported about environment outcomes and the effects on human health resulted from the exposure to those substances [1, 5-10]. Those studies described potential estrogenic and carcinogenic activity of parabens, which classifies them as endocrine disrupting chemicals (EDCs).

Although parabens are still used, mainly in cosmetic products, the concern regarding the adverse effects on human health has led to a gradually restriction on their usage. Besides the fact that most countries do not have regulations to parabens usage, several organizations, such as US Food and Drug Administration (FDA), World Health Organization (WHO), European Union (EU) and Scientific Society on Costumer Safety (SCCS), have stablished low concentration limits of those compounds in the case of some types of products. For instance, the content of ethyl and butyl parabens in cosmetics was changed from $0.4 \%$ to $0.14 \%$ in all EU members in 2014, and these values were also applied in several South America countries [10-12]. In this context, several industries have been replacing parabens to other preservatives with a police of "paraben-free" products. However, parabens are still largely detected in various environmental matrices [12-15], which has led to the contamination of potable water and increased the exposure of humans to those compounds.

In order to remove emerging contaminants, such as parabens, from water and wastewater, the scientific community has invested in the development and enhancement of advanced oxidation processes (AOPs) [16-18]. These processes are based on the generation of hydroxyl radicals $\left({ }^{\bullet} \mathrm{OH}\right)$, which are highly reactive $\left(\mathrm{E}^{0}=2.8 \mathrm{~V}\right)$ and promote the degradation of organic pollutants. Fenton process, photolysis, ozonation and other AOPs are very efficient for the removal of certain pollutants, however not necessarily effect for the complete depletion of organic matter on the treatment of more recalcitrant substances. For that purpose, electrochemical AOPs have shown promising results on the total removal of organic contaminants from water [19-21]. For instance, Wang et al. [22], Dantas et al. [23], Li et al. [24] have studied the degradation of the antibiotic sulfamethoxazole (SMX) by Fenton, 
ozonation and electrochemical processes, respectively, and found that total SMX can be removed by the applied treatments. However, removal of TOC (total organic carbon) is different for each case: with Fenton reaction $83 \%$ of TOC was removed, ozonation promoted only $10 \%$ of TOC removal and electrochemical process achieved $91 \%$ of mineralization. Additionally, Amorim et al. [25] studied electrochemical degradation of SMX in the presence of trimethoprim, being both pollutant completely removed and $90 \%$ of TOC reduced.

On electrochemical process, organic compounds can be directly oxidized by electron transfer on the surface of the electrode, or indirectly oxidized by ${ }^{\bullet} \mathrm{OH}$ or other oxidizing agents. In the second case, oxidants are electrochemically produced on the anode and can react with organic compounds at the mediations of the electrode or extend the oxidation to the bulk of the solution. In most scenarios both oxidation mechanisms simultaneously occur and can be explored according to the objective of the treatment. Most recently, researchers have been exploring the coupling of technologies, in order to improve the efficiency of electrochemical treatments. Electro-Fenton process [21, 26], electrolysis irradiated by UV light [27-29] and electrochemistry combined to sonochemistry [30-32] can be highlighted, where in most cases better results are observed for the coupled processes due to a synergistic effect. As a result of the coupling, more oxidant agents can be produced and, most important, it can be promoted the activation of such species, which means that highly reactive species can be formed from poorly reactive oxidants.

Sonochemical processes are based on the irradiation of ultrasound through an aqueous medium. The resultant effect is the so-called cavitation phenomena, which consists on the formation, expansion and implosion of bubbles. The energy released in this cavitation process is the principle of sonochemistry, in which the most accepted theory states that at the point of bubble implosion, conditions of thousands of kelvins and hundreds of atm are produced (hot-spot theory). This conditions are very short-lived but sufficient to promote the generation of radicals, such as hydroxyl $\left({ }^{\bullet} \mathrm{OH}\right)$, hydrogen $\left({ }^{\bullet} \mathrm{H}\right)$ and hydroperoxyl $\left(\mathrm{HO}_{2}{ }^{\bullet}\right)$ [33-35]. Coupling sonochemical to electrochemical technology it is possible to improve the formation of radicals, activate oxidants and also favor the mass-transfer process [30, 36, 37].

However, ultrasound irradiation as a wastewater treatment is still considered an emergent technology and more need to be known about frequency and power effects, $\mathrm{pH}$, influence of complex matrices and set-up of the sonolytic system used [38, 39]. According to that, this study aims to enhance electrolysis by the coupling of ultrasound, but more importantly, it focuses on the understanding of cavitation effect on electrogenerated oxidant species. For that, the 
degradation of methyl paraben, as a model molecule, was carried out under the presence of different supporting electrolytes and different conditions of ultrasound frequency.

\section{Materials and methods}

\section{Chemicals}

All solutions were prepared using high-purity water obtained from a Millipore Milli-Q system (resistivity $>18 \mathrm{M} \mathrm{cm}$ at $25^{\circ} \mathrm{C}$ ). Methyl paraben and the reactants for determination of oxidants (KI, $\mathrm{Na}_{2} \mathrm{~S}_{2} \mathrm{O}_{3}, \mathrm{NaOH}, \mathrm{As}_{2} \mathrm{O}_{3}$ and starch solution (1\%)) were purchased from Sigma-Aldrich. Supporting electrolytes $\mathrm{Na}_{2} \mathrm{SO}_{4}$ and $\mathrm{NaCl}$ were obtained from Panreac, as well as, $\mathrm{H}_{2} \mathrm{SO}_{4}$ used for $\mathrm{pH}$ adjustment. Acetonitrile, from VWR Chemicals, was used as mobile phase for HPLC analysis. All reactants were used as received.

\section{Experimental set-up}

Experiments were carried out in a bench scale flow system operating in a discontinuous mode. Electrochemical reactor is coupled to a peristaltic pump (PERCOM-I, JP Selecta, Barcelona, Spain) and to a jacketed glass vessel, used to store $1 \mathrm{~L}$ of solution. The cell is equipped with a plate of boron doped diamond (BDD) as anode and a plate of stainless steel as cathode (both with surface area of $75 \mathrm{~cm}^{-2}$ ) and a HQ-Power PS3010 power supply (0-30 V, 0-10 A) provided the applied current. A thermostatic bath (Digiterm 100, JP Selecta) was used to maintain the solution temperature at $25^{\circ} \mathrm{C}$. Tests using ultrasound were carried out at three different acoustic frequencies: $20 \mathrm{kHz}$ (UP200S, Hielscher Ultrasonics GmbH), $1 \mathrm{MHz}$ and $10 \mathrm{MHz}$ (EPOCH 650 , Olympus). The ultrasound probe was immersed in the glass tank directly acting in the working solution.

\section{Analytical techniques}

High performance liquid chromatography (Agilent Technologies 1260 chromatographer) was used to monitor the concentration of methyl paraben with an Eclipse Plus C18 column $(3.5 \mu \mathrm{m}$ $4.6 \times 100 \mathrm{~mm}$ ), at $25^{\circ} \mathrm{C}$, and UV detector set in $280 \mathrm{~nm}$. Mobile phase was acetonitrile and water $(60: 40 \mathrm{v} / \mathrm{v})$ with $1 \mathrm{~mL} \mathrm{~min}^{-1}$ of flow rate. Total organic carbon (TOC) was determined in a Multi N/C 3100 Analytik Jena analyzer. Measurements of $\mathrm{pH}$ and conductivity were carried out in a GLP22 CRISON and GLP 31 CRISON, respectively. Oxidizing power of the solution was determined by iodometric titration. Inorganic anions $\left(\mathrm{Cl}^{-}, \mathrm{ClO}_{3}{ }^{-}\right.$and $\left.\mathrm{ClO}_{4}{ }^{-}\right)$were measured by ion chromatography using a 930 Compact IC Flex (Metrohm) and hypochlorite was determined by titration, with a solution of $\mathrm{As}_{2} \mathrm{O}_{3}$ in $2.0 \mathrm{M} \mathrm{NaOH}$. 
142 Synergistic index (SI), Eq. 1, was used to evaluate the effect of coupling ultrasound to 143 electrolysis. Parameters $\mathrm{k}_{\mathrm{p} 1-\mathrm{p} 2}, \mathrm{k}_{\mathrm{p} 1}$ and $\mathrm{k}_{\mathrm{p} 2}$ are the kinetic constants for the coupled process and 144 the individual processes 1 and 2, respectively.

$145 \quad S I=\frac{k_{p 1-p 2}}{k_{p 1}+k_{p 2}}$

147 Results and discussion

148 Fig. 1 shows the removal of MeP obtained in sulfate and chloride medium by the individual 149 and coupled processes. Sonochemical process (SCh) showed a very low efficiency to the 150 oxidation of $\mathrm{MeP}$, achieving 26 and $30 \%$ of removal in chloride and sulfate media, 151 respectively. The propagation of ultrasound in a liquid occurs by the vibration of the molecules 152 in the medium, which produces small variations of pressures and cycles of alternate expansions 153 and compressions [40]. In the case of water saturated with air, the acoustic cavitation effect 154 will be produced, creating favored conditions to the formation of radical oxidants [33, 39].

155 Under the studied conditions it is expected the formation of $\bullet$ OH, $\mathrm{H}_{2} \mathrm{O}_{2}$ and $\mathrm{O}_{3}$ from water 156 sonolysis according to the Eqs. 2-9 (where the symbol ))) represents the ultrasound waves). 157 Moreover, in chloride medium, it is also possible the formation of active chlorine species (Eqs. 158 10-12) $[35,41]$. Hence, the faster MeP removal observed in chloride medium, for SCh process, 159 can be explained by the higher concentration of oxidants and the rapid production of 160 organochlorinated compounds (characteristic of oxidation by chlorine species).

$2^{\bullet} \mathrm{OH} \rightarrow \mathrm{H}_{2} \mathrm{O}_{2}$

$\mathrm{H}^{\bullet}+\mathrm{O}_{2} \rightarrow \mathrm{HO}_{2}^{\bullet}$

$\mathrm{HO}_{2}^{\bullet}+\mathrm{HO}_{2}^{\bullet} \rightarrow \mathrm{H}_{2} \mathrm{O}_{2}+\mathrm{O}_{2}$

$\mathrm{H}^{\bullet}+\mathrm{HO}_{2}^{\bullet} \rightarrow \mathrm{H}_{2} \mathrm{O}_{2}$

$\mathrm{O}^{\bullet}+\mathrm{O}_{2} \rightarrow \mathrm{O}_{3}$

$\mathrm{H}^{\bullet}+\mathrm{O}_{2} \rightarrow{ }^{\bullet} \mathrm{OH}+\mathrm{O}^{\bullet}$

$\left.\left.\left.169 \mathrm{Cl}^{-}+\right)\right)\right) \rightarrow \mathrm{Cl}_{2}$ 
172 Electrochemical processes, individually (ECh) and coupled to sonolysis (SECh), completely 173 removed MeP. However, under the applied conditions, it was not possible to observe an 174 improvement on the efficiency for the coupled process. Different characteristics for the MeP 175 degradation in sulfate and chloride media were expected, as observed for diamond electrolysis 176 in a previous work [27]. The main species generated in each system are $\mathrm{SO}_{4}^{-\bullet}$ and $\mathrm{ClO}^{-}$, which 177 results in different mechanism of oxidation. Sulfate radical has a redox potential comparable 178 to that of ${ }^{\bullet} \mathrm{OH}$ (2.6 and $2.8 \mathrm{~V}$ vs. normal hydrogen electrode, NHE, respectively) and its main 179 oxidation mechanism is by electron transfer, which promotes the formation of organic radicals 180 and its further rupture of bonds, resulting in high elimination of organic matter $[42,43]$. On the other hand, hypochlorite anions will rapidly convert the pollutant into organochlorinated compouds by the addition of $\mathrm{Cl}$ atoms to its structure [44].

Although no improvement was observed for MeP removal, the SECh process accelerated the oxidation of total organic matter, as it is shown in Fig. 2 and Tab. 1. Two kinetic regions of pseudo-first order can be observed in the case of electrolytic processes, with $\mathrm{k}_{1}$ and $\mathrm{k}_{2}$ as the kinetic constants for each region. This transition is explained by the nature of the generated intermediates, which seem to be easily oxidized by the present oxidant agents than the initial pollutant [27]. No removal of TOC was achieved with SCh during the 8 hours of treatment (the negative value of kinetic constant is attributed to the dispersion of the points), but complete mineralization was attained for ECh and SECh in less than 5 hours. The coupled process showed an improvement on the oxidation of organic matter due to the sonochemical activation of oxidants. In sulfate medium, $\mathrm{SO}_{4}^{-\bullet}$ is produced by the homogeneous cleavage of the electrogenerated persulfate (Eq. 13), which is more efficient than persulfate itself.

194 Additionally, the radicals produced by water sonolysis can also react with persulfate and contribute to radical sulfate yield (Eqs. 14 and 15) [45, 46].

$\mathrm{S}_{2} \mathrm{O}_{8}^{-2}+{ }^{\bullet} \mathrm{OH} \rightarrow 2 \mathrm{HSO}_{4}^{-}+\left(\mathrm{SO}_{4}^{-}\right)^{\bullet}+1 / 2 \mathrm{O}_{2}$

$\mathrm{S}_{2} \mathrm{O}_{8}{ }^{-2}+{ }^{\bullet} \mathrm{H} \rightarrow \mathrm{HSO}_{4}^{-}+\left(\mathrm{SO}_{4}^{-}\right)^{\bullet}$

On the other hand, an interesting behavior is observed for chloride medium: in a first moment, 
201

202

203

204

205

206

207

208

209

210

211

212

213

214

215

216

217

218

219

220

221

222

223

224

225

226

227

228

229

230

231

232

In order to better understand this result, it is necessary to take into account the nature of oxidants species formed and the possible effect of ultrasound on it. It is well known that in an electrochemical bath, chloride anions are oxidized to chlorine gas. As it was mentioned, the effects of ultrasound are related to the gas dissolved into the water [34, 41, 46]. Higher concentration of gases, may improve the cavitation effect, however after a certain time the solution will be saturated and this improvement will decrease its significance. In addition to this, ultrasound can promote the formation of $\mathrm{Cl}^{\bullet}$ and $\mathrm{Cl}_{2}{ }^{\bullet}$ species (Eq. 16 and 17), which are strong oxidants for many organic compounds [47]. The slower rate in the second kinetic region could be attributed to the nature of the intermediates formed, which can be less reactive with relation to the oxidants present in the medium.

$\left.\left.\left.\mathrm{Cl}_{2}+\right)\right)\right) \rightarrow 2 \mathrm{Cl}^{\bullet}$

$\mathrm{Cl}^{\bullet}+\mathrm{Cl}^{-} \rightleftharpoons \mathrm{Cl}_{2}^{-\bullet}$

Considering that two regions of kinetics can be observed, it is not possible to determine one synergistic index (SI) for all the treatment (inset of Fig. 2). In a first moment chloride medium is better, with an SI 4.5 times higher than sulfate. On the other hand, for the second region, sulfate medium presented an SI 5.6 times higher than chloride. These results are important to guess the conditions in which ultrasound may have more influence and this can be an important step for future studies, where an optimization of those conditions could be evaluated.

It is well-known and described the formation of chlorate and perchlorate in electrolysis of chlorine using BDD electrodes (Eqs. 18-21) [21, 48, 49]. In addition to the fact that those species have a lower oxidant potential, which decrease the process efficiency, they are also related to serious health problems and, thus, are not desired to be produced. Fig. 3 shows the speciation of the oxochloranions detect in ECh and SECh processes. Although no formation of oxochloranions were verified for sonolysis, it is possible to observe a decrease of the chloride concentration, which is attributed to the formation of $\mathrm{Cl}_{2}$ and in agreement with the results and discussion of Fig. 1. The behavior of production / consumption of chlorine species are similar for ECh and SCEh; however, it is important to note that the coupling of ultrasound delays the formation of perchlorate and that this only starts after all TOC was removed, at 4 hours of treatment (Fig. 2). Hence, considering the batch system studied, seems feasible the application of electrochemical process coupled to ultrasound during 4 hours, minimizing the adverse production of chlorate and perchlorate.

$\mathrm{Cl}^{-}+{ }^{\bullet} \mathrm{OH} \rightarrow \mathrm{ClO}^{-}+\mathrm{H}^{+}+\mathrm{e}^{-}$ 
236 Considering that the coupling of sonolysis to electrochemical treatment showed faster 237 mineralization of organic matter with a decrease on the formation of chlorate species, it was investigated the effect of ultrasound frequency on the system. The frequency applied has an important role on the critical size of the bubbles and, thus, on the cavitation phenomena. Three acoustic frequencies were studied: $20 \mathrm{kHz}$ (power ultrasound), with $20 \%$ and $75 \%$ of power (conditions referred as L20 and L75), $1 \mathrm{MHz}$ (high frequency ultrasound) (referred as H1) and $10 \mathrm{MHz}$ (diagnostic ultrasound) (referred as H10).

Fig. 4 shows the removal of MeP and TOC obtained for SECh process. Single sonolytic process resulted in low removal of MeP and none removal of TOC. Negative values observed for 10 MHz (Fig. 4a) are attributed to error of the method, meaning that no removal was achieved under this condition. Lower frequency showed better MeP removals: at $20 \mathrm{kHz}$ approximately $30 \%$ was attained for both power percentages, while at $1 \mathrm{MHz}$ only $7 \%$ was removed. SECh treatment seems to slightly enhance ECh results, however it was not possible to observe great variations of efficiency for different frequencies, as it was expected. Frequency does not seem to affect the MeP removal (Fig. 4a), in which total oxidation is achieved with approximately 4 hours for all electrochemical processes. However, at the second kinetic region of TOC removal (Fig. 4b), it is possible to observe different rates: at L75 and at H1, complete mineralization is attained with 3 hours, while for other conditions 4 hours were necessary. Moreover, the kinetic constant calculated for L75 is 1.5 times higher than for $\mathrm{H} 1$ (1.42 compared to $\left.0.93 \mathrm{~h}^{-1}\right)$.

As it was already stated, ultrasound will directly influence the gas dissolved in the working solution, because of the cavitation phenomena. Cavitation bubbles have three sites in which chemical reactions can take place: inside the bubble, where temperatures are very high and thermal reactions can occur; the gas-liquid interface, in which hydrophobic compounds are more concentrated; and the bulk of the solution $[39,50,51]$. If volatile intermediates are produced, they can be oxidized inside the bubbles and, thus, the effect of the ultrasound will be more remarkable. As a matter of fact, phenol is a typical product of the degradation of MeP [52] and that is the main reason why the differences between applied frequencies can only be seen in the second region of TOC kinetics. 
Synergistic index was calculated for each applied frequency in terms of MeP removal and TOC mineralization (Fig. 5). First, it is important to highlight that SI does not consider the time needed to remove all MeP or organic matter. This means that although SI is higher for L20 and H10 conditions, at L75 and H1 complete mineralization was achieved 1 hour faster than in the other conditions. Hence the order of efficiency can be summarized as L75 > H1 > L20 > H10. These results indicate that there is an optimal ultrasound frequency in which the degradation is favored, as it was previous observed in the literature [53-55].

At lower frequencies, ultrasound promotes the so-called transient cavitation, in which the bubbles are very unstable and the collapses are more violent, leading to sites of high temperature and pressure. At this point, it can be expected high production of radicals by sonolysis and the activation of oxidants. On the other hand, at high frequencies, the cavitation bubbles are stable and its collapse is less violent. Besides the lower cavitation intensity, radicals are still produced inside the bubbles, and its longer stability permits that those radicals escape to the bulk solution and react with organic compounds [34, 35, 53]. It is estimated that under transient cavitation, $10 \%$ of the radicals escape to the bulk solution; under stable cavitation this percentage could be improved [34]. Therefore, the optimal frequency will be a balance between the effects of transient and stable cavitation. It is plausible to consider that under the applied conditions, the high frequencies effect compensates the low frequencies one and thus, a very small difference is observed in the final result.

As an attempt to clarify the role of ultrasound on the mediated oxidation, sonochemical tests were carried out in the presence of persulfate at the different frequencies. For that, $12.5 \mathrm{~g} \mathrm{~L}^{-1}$ of sodium persulfate were added to the synthetic wastewater studied before $\left(100 \mathrm{mg} \mathrm{L}^{-1}\right.$ of $\mathrm{MeP}+3.0 \mathrm{~g} \mathrm{~L}^{-1}$ of $\mathrm{Na}_{2} \mathrm{SO}_{4}$ ) and ultrasound was applied for 8 hours, point in which the concentration of MeP and TOC were measured. Tab. 2 shows that persulfate itself is capable to remove approximately $60 \%$ of $\mathrm{MeP}$ and $35 \%$ of organic matter. The irradiation of low frequency ultrasound enhances the oxidation and almost total mineralization is achieved. In the absence of ultrasound, persulfate is the only oxidant in the medium; on the other hand, in the sonolytic process ${ }^{\bullet} \mathrm{OH}$ is generated and $\mathrm{SO}_{4}{ }^{\bullet}$ is produced by the activation of persulfate, as it was already discussed. However, for $1 \mathrm{MHz}$ and $10 \mathrm{MHz}$, the efficiency of the process significantly decreases compared to persulfate alone. This result suggests that, under these conditions, ultrasound is not only not activating the persulfate, but also inhibiting this oxidant, which could be explained by Eqs. 22 and 23 [56]. Water sonolysis occurs even at high frequencies, thus, hydrogen peroxide is produced from the recombination of radicals (Eqs. 4, 
6 and 7) and will react with persulfate. Additionally, even though the main source of sulfate radical is suppressed (persulfate sonolysis, Eq. 13), it may still be produced, at smaller concentrations, by the reaction of persulfate with ${ }^{\bullet} \mathrm{OH}$ and ${ }^{\bullet} \mathrm{H}$ (Eqs. 14 and 15); however, they can also be consumed by $\mathrm{H}_{2} \mathrm{O}_{2}$.

$\mathrm{S}_{2} \mathrm{O}_{8}{ }^{-2}+\rightarrow 2 \mathrm{H}^{+}+2 \mathrm{SO}_{4}{ }^{2-}+\mathrm{O}_{2}$

303 In conclusion, low frequency ultrasound (transient cavitation) activates persulfate, producing $\mathrm{SO}_{4}{ }^{\bullet}$, that in addition to ${ }^{\bullet} \mathrm{OH}$, from water sonolysis, improve organic matter degradation. On the other hand, high frequency ultrasound (stable cavitation) is not able to activate persulfate and also can inhibit its action by the $\mathrm{H}_{2} \mathrm{O}_{2}$ produced from water sonolysis. These is in agreement with the observations of Ferkous et al. [45], which studied the persulfate oxidation of naphthol blue black enhanced by ultrasound and stated that persulfate-enhanced effect was more remarkable at $20 \mathrm{kHz}$ than at $585 \mathrm{kHz}$. In addition, most studies of ultrasound activation of persulfate only studied low frequency, mainly $20 \mathrm{kHz}$, obtaining very promising results [57$60]$.

At this point, it is important to remember that electrochemical cell behaves as a cocktail of species and not only persulfate and hydroxyl radicals are acting; all species can suffer the effects of violent and/or stable cavitation in different ways. Irradiation of ultrasound will not only produce more oxidants, but also influences on the kinetics of all reactions, increasing or decreasing their kinetic rates. Furthermore, physical effects can take place other than the chemical ones. For instance, violent cavitation, in transient regime, can also improve the masstransport of species, which will improve the electrochemical process. These points reinforce the theory that the optimal ultrasound frequency to enhance an electrochemical system will be a balance between the effects of transient and stable cavitation, which represents a combination of all factors involved. Although it was expected higher improvement for the coupled process and greater differences between the applied frequencies, the results presented and discussed give an essential indication of how ultrasound acts in the studied system. It is also important to take into account that the system studied is simple and MeP is a model molecule, with a relatively easy oxidation.

\section{Conclusions}


The coupling of ultrasound to electrolysis was studied for the degradation of methyl paraben synthetic wastewater, where the influence of supporting electrolyte and acoustic frequencies were evaluated. From that, the following conclusions can be drawn:

- The target pollutant was completely mineralized by single electrolysis with diamond anodes and coupled to ultrasound. However, while removal of MeP is faster in the presence of chloride, its mineralization is more efficient in sulfate medium, due to the nature of the oxidant species generated in each condition.

- The mineralization of the wastewater by single electrolysis and coupled to ultrasound fits well to pseudo-first order kinetics, although two regions can be distinguished. The transition point between regions and the relation between $\mathrm{k}_{1}$ and $\mathrm{k}_{2}$ will depend on the reactivity of the intermediates formed and the nature of the main oxidant specie in the medium.

- The combination of electrolysis and sonolysis has a synergistic effect on the abatement of organic matter, in both chloride and sulfate media, due to the improvement on mediated $341 \quad$ oxidation.

- Ultrasound promotes different effects of cavitation at low and high frequencies. Hence,

343 there is an optimal frequency for different systems, in which those effects are balanced. It

344 is possible that, under specific conditions, the high frequencies effect compensates the low

345 frequencies one and thus, a very small difference is observed in the final result.

- Sonochemical tests showed that low frequency ultrasound can activate persulfate oxidants

347 to form radical species, which highly improves the oxidation. However, it also suggests that

348 high frequencies are not efficient on the activation of persulfate and also inhibit its oxidative 349 action by converting it into sulfate anions. 

source of exposure to parabens in the U.S. population, Environmental Research, 164 (2018) 580-584.

[2] M.G. Soni, S.L. Taylor, N.A. Greenberg, G.A. Burdock, Evaluation of the health aspects of methyl paraben: a review of the published literature, Food and Chemical Toxicology, 40 (2002) 1335-1373.

358 [3] P.D. Darbre, A. Aljarrah, W.R. Miller, N.G. Coldham, M.J. Sauer, G.S. Pope, Concentrations of parabens in human breast tumours, Journal of Applied Toxicology, 24 (2004) 5-13. [4] Z. Dagher, M. Borgie, J. Magdalou, R. Chahine, H. Greige-Gerges, p-Hydroxybenzoate esters metabolism in MCF7 breast cancer cells, Food and Chemical Toxicology, 50 (2012) 4109-4114.

[5] M.G. Soni, I.G. Carabin, G.A. Burdock, Safety assessment of esters of p-hydroxybenzoic acid (parabens), Food and Chemical Toxicology, 43 (2005) 985-1015.

[6] J. Boberg, C. Taxvig, S. Christiansen, U. Hass, Possible endocrine disrupting effects of parabens and their metabolites, Reproductive Toxicology, 30 (2010) 301-312.

[7] N. Aubert, T. Ameller, J.-J. Legrand, Systemic exposure to parabens: Pharmacokinetics, tissue distribution, excretion balance and plasma metabolites of [14C]-methyl-, propyl- and butylparaben in rats after oral, topical or subcutaneous administration, Food and Chemical Toxicology, 50 (2012) 445-454.

[8] D. Bledzka, J. Gromadzinska, W. Wasowicz, Parabens. From environmental studies to human health, Environment International, 67 (2014) 27-42.

[9] J.C. Xue, K. Kannan, Accumulation profiles of parabens and their metabolites in fish, black bear, and birds, including bald eagles and albatrosses, Environment International, 94 (2016) 546-553.

[10] K. Nowak, W. Ratajczak-Wrona, M. Górska, E. Jabłońska, Parabens and their effects on the endocrine system, Molecular and Cellular Endocrinology, (2018).

378 [11] T. Velegraki, E. Hapeshi, D. Fatta-Kassinos, I. Poulios, Solar-induced heterogeneous photocatalytic degradation of methyl-paraben, Applied Catalysis B-Environmental, 178 (2015) 2-11.

[12] A.V. Marta-Sanchez, S.S. Caldas, A. Schneider, S.M.V.S. Cardoso, E.G. Primel, Trace analysis of parabens preservatives in drinking water treatment sludge, treated, and mineral water samples, Environmental Science and Pollution Research, (2018).

[13] W.H. Li, Y.L. Shi, L.H. Gao, J.M. Liu, Y.Q. Cai, Occurrence, fate and risk assessment of parabens and their chlorinated derivatives in an advanced wastewater treatment plant, Journal of Hazardous Materials, 300 (2015) 29-38. [14] J. Chen, B.F.G. Pycke, B.J. Brownawell, C.A. Kinney, E.T. Furlong, D.W. Kolpin, R.U. Halden, Occurrence, temporal variation, and estrogenic burden of five parabens in sewage sludge collected across the United States, Science of the Total Environment, 593 (2017) 368-374. countries, Greece, and the United States, Chemosphere, 201 (2018) 13-19.

[16] J.L. Wang, S.Z. Wang, Removal of pharmaceuticals and personal care products (PPCPs) from wastewater: A review, Journal of Environmental Management, 182 (2016) 620-640. Formulations, Effects, Fate, InTech, Rijeka, 2011, pp. 808. 

aqueous systems by advanced oxidation processes, Environment International, 35 (2009) 402-417.

[19] I. Sires, E. Brillas, M.A. Oturan, M.A. Rodrigo, M. Panizza, Electrochemical advanced oxidation processes: today and tomorrow. A review, Environmental Science and Pollution Research, 21 (2014) 8336-8367. [20] H. Sarkka, A. Bhatnagar, M. Sillanpaa, Recent developments of electro-oxidation in water treatment - A review, Journal of Electroanalytical Chemistry, 754 (2015) 46-56. [21] F.C. Moreira, R.A.R. Boaventura, E. Brillas, V.J.P. Vilar, Electrochemical advanced oxidation processes: A review on their application to synthetic and real wastewaters, Applied Catalysis B-Environmental, 202 (2017) 217-261.

[22] S.Z. Wang, J.L. Wang, Comparative study on sulfamethoxazole degradation by Fenton and Fe(II)-activated persulfate process, Rsc Advances, 7 (2017) 48670-48677.

410 [23] R.F. Dantas, S. Contreras, C. Sans, S. Esplugas, Sulfamethoxazole abatement by means of ozonation, Journal of Hazardous Materials, 150 (2008) 790-794.

[24] S. Li, D. Bejan, M.S. McDowell, N.J. Bunce, Mixed first and zero order kinetics in the electrooxidation of sulfamethoxazole at a boron-doped diamond (BDD) anode, Journal of Applied Electrochemistry, 38 (2008) 151-159.

[25] K.P. de Amorim, L.L. Romualdo, L.S. Andrade, Electrochemical degradation of sulfamethoxazole and trimethoprim at boron-doped diamond electrode: Performance, kinetics and reaction pathway, Separation and Purification Technology, 120 (2013) 319-327. [26] R.C. Burgos-Castillo, I. Sires, M. Sillanpaa, E. Brillas, Application of electrochemical advanced oxidation to bisphenol A degradation in water. Effect of sulfate and chloride ions, Chemosphere, 194 (2018) 812-820.

[27] D. Dionisio, A.J. Motheo, C. Sáez, M.A. Rodrigo, Effect of the electrolyte on the electrolysis and photoelectrolysis of synthetic methyl paraben polluted wastewater, Separation and Purification Technology, (2018).

424 [28] J.M. Aquino, D.W. Miwa, M.A. Rodrigo, A.J. Motheo, Treatment of actual effluents produced in the manufacturing of atrazine by a photo-electrolytic process, Chemosphere, 172 (2017) 185-192.

[29] S. Hussain, J.R. Steter, S. Gul, A.J. Motheo, Photo-assisted electrochemical degradation of sulfamethoxazole using a Ti/Ru0.3Ti0.7O2 anode: Mechanistic and kinetic features of the process, Journal of Environmental Management, 201 (2017) 153-162.

[30] J.R. Steter, D. Dionisio, M.R.V. Lanza, A.J. Motheo, Electrochemical and sonoelectrochemical processes applied to the degradation of the endocrine disruptor methyl paraben, Journal of Applied Electrochemistry, 44 (2014) 1317-1325.

[31] F.L. Souza, C. Saez, P. Canizares, A.J. Motheo, M.A. Rodrigo, Coupling photo and sono technologies to improve efficiencies in conductive diamond electrochemical oxidation, Applied Catalysis B-Environmental, 144 (2014) 121-128.

436 [32] J.R. Steter, M.H. Kossuga, A.J. Motheo, Mechanistic proposal for the electrochemical and sonoelectrochemical oxidation of thiram on a boron-doped diamond anode, Ultrasonics Sonochemistry, 28 (2016) 21-30.

[33] Y.G. Adewuyi, Sonochemistry: Environmental science and engineering applications, Industrial \& Engineering Chemistry Research, 40 (2001) 4681-4715.

441 [34] L.H. Thompson, L.K. Doraiswamy, Sonochemistry: Science and engineering, Industrial \& 442 Engineering Chemistry Research, 38 (1999) 1215-1249. 

in Ultrasound Technology for Environmental Remediation, Springer Netherlands, Dordrecht, 2013, pp. 5-12. [36] G.S. Garbellini, G.R. Salazar-Banda, L.A. Avaca, Aplicação do ultra-som em sistemas eletroquímicos: considerações teóricas e experimentais, Química Nova, 31 (2008) 123-133. [37] M.J.M. de Vidales, S. Barba, C. Saez, P. Canizares, M.A. Rodrigo, Coupling ultraviolet light and ultrasound irradiation with Conductive-Diamond Electrochemical Oxidation for the removal of progesterone, Electrochimica Acta, 140 (2014) 20-26. oxidation processes based on cavitation - A review, Chemical Engineering Journal, 338 (2018) 599-627.

[39] J. Lifka, B. Ondruschka, J. Hofmann, The Use of Ultrasound for the Degradation of Pollutants in Water: Aquasonolysis - A Review, Engineering in Life Sciences, 3 (2003) 253-262. [40] A. Weissler, H.W. Cooper, S. Snyder, Chemical effect of ultrasonic waves - oxidation of potassium iodide solution by carbon tetrachloride, Journal of the American Chemical Society, 72 (1950) 1769-1775. Involving Inorganic Systems, Springer Netherlands, Dordrecht, 2011, pp. 213-271.

461

462

[42] H. Lutze, Sulfate radical based oxidation in water treatment, Institut für Instrumentelle Analytische Chemie, Universität Duisburg-Essen, Essen, Germany, 2013, pp. 163.

[43] Y. Yang, J. Jiang, X.L. Lu, J. Ma, Y.Z. Liu, Production of Sulfate Radical and Hydroxyl Radical by Reaction of Ozone with Peroxymonosulfate: A Novel Advanced Oxidation Process, Environmental Science \& Technology, 49 (2015) 7330-7339.

[44] M. Mascia, A. Vacca, S. Palmas, Fixed bed reactors with three dimensional electrodes for electrochemical treatment of waters for disinfection, Chemical Engineering Journal, 211 (2012) 479-487. degradation of naphthol blue black in water: Evidence of sulfate radical formation, Ultrasonics Sonochemistry, 34 (2017) 580-587. persulfate activated by different methods for PAHs removal in soil, Journal of Hazardous Materials, 254 (2013) 228-235. Gonzalez, Kinetic study of the reactions of chlorine atoms and $\mathrm{Cl} 2$ - radical anions in aqueous solutions. 1. Reaction with benzene, The Journal of Physical Chemistry A, 104 (2000) 478 3117-3125.

479 [48] C.d.N. Brito, D.M. de Araújo, C.A. Martínez-Huitle, M.A. Rodrigo, Understanding active chlorine species production using boron doped diamond films with lower and higher sp3/sp2 ratio, Electrochemistry Communications, 55 (2015) 34-38. Formation on Boron-Doped Diamond Film Anodes, Environmental Science \& Technology, 45 (2011) 10582-10590. sonochemical degradation of monocyclic aromatic compounds is aqueous solution, Journal of Physical Chemistry, 100 (1996) 11636-11642. 
[51] P. Chowdhury, T. Viraraghavan, Sonochemical degradation of chlorinated organic compounds, phenolic compounds and organic dyes - A review, Science of the Total Environment, 407 (2009) 2474-2492.

491 [52] J.R. Steter, R.S. Rocha, D. Dionisio, M.R.V. Lanza, A.J. Motheo, Electrochemical oxidation 492 route of methyl paraben on a boron-doped diamond anode, Electrochimica Acta, 117 (2014) 127-133.

494 [53] K. Kim, E. Cho, B. Thokchom, M. Cui, M. Jang, J. Khim, Synergistic sonoelectrochemical 495 removal of substituted phenols: Implications of ultrasonic parameters and physicochemical 496 properties, Ultrasonics Sonochemistry, 24 (2015) 172-177.

497 [54] M. Capocelli, E. Joyce, A. Lancia, T.J. Mason, D. Musmarra, M. Prisciandaro, Sonochemical 498 degradation of estradiols: Incidence of ultrasonic frequency, Chemical Engineering Journal, $499210(2012) 9-17$.

500 [55] J.W. Kang, H.M. Hung, A. Lin, M.R. Hoffmann, Sonolytic destruction of methyl tert-butyl 501 ether by ultrasonic irradiation: The role of $\mathrm{O}-3, \mathrm{H} 2 \mathrm{O} 2$, frequency, and power density, 502 Environmental Science \& Technology, 33 (1999) 3199-3205.

503 [56] Y. Yang, H.G. Guo, Y.L. Zhang, Q.Z. Deng, J. Zhang, Degradation of Bisphenol A Using 504 Ozone/Persulfate Process: Kinetics and Mechanism, Water Air and Soil Pollution, 227 (2016). 505 [57] Z.S. Wei, F.A. Villamena, L.K. Weavers, Kinetics and Mechanism of Ultrasonic Activation 506 of Persulfate: An in Situ EPR Spin Trapping Study, Environmental Science \& Technology, 51 507 (2017) 3410-3417.

508 [58] C.H. Weng, K.L. Tsai, Ultrasound and heat enhanced persulfate oxidation activated with 509 Fe-0 aggregate for the decolorization of $\mathrm{Cl}$ Direct Red 23, Ultrasonics Sonochemistry, 29 $510 \quad$ (2016) 11-18.

511 [59] C. Cai, H. Zhang, X. Zhong, L.W. Hou, Ultrasound enhanced heterogeneous activation of 512 peroxymonosulfate by a bimetallic Fe-Co/SBA-15 catalyst for the degradation of Orange II in 513 water, Journal of Hazardous Materials, 283 (2015) 70-79.

514 [60] L.W. Hou, H. Zhang, X.F. Xue, Ultrasound enhanced heterogeneous activation of 515 peroxydisulfate by magnetite catalyst for the degradation of tetracycline in water, Separation and Purification Technology, 84 (2012) 147-152. 